\title{
Interaction between Allocentric and Egocentric Space Representations in Mathematical Model of Neurodynamics Underlying Spatial Cognition
}

\author{
Kharybina Zoya \\ Southern Federal University \\ Rostov-on-Don, Russia
}

\begin{abstract}
Navigation in space requires different sources of spatial information. The paper presents biologically inspired mathematical model of spatial navigation. In the model, spatial representation is assumed to result from the interaction between allocentric and egocentric representations, based on the information about environmental borders and integration of selfmotion velocity, respectively. It is proposed that the same interaction should underlie spatial representation in rodent hippocampus.
\end{abstract}

Keywords- Allocentric Representation; Egocentric Representation; Place Cells; Cognitive Map; Grid Cells; Border Cells

\section{INTRODUCTION}

During navigational behavior animals compose inner mental map of physical space, called cognitive map. This map is linked to the activity of hippocampal place cells [1]. Place cell is active when the animal enters a small field within a spatial environment and is silent outside this location. Receptive field of place cell, or place field, is unique for a given environment.

To compose a cognitive map animals use various sources of information that can be divided into inner and external. The first group is presented by sensor flows generated during locomotion (proprioceptive, tactile, olfactory and other modalities); second one includes physical field gradients and distal cues used to obtain directional information and local cues used to correct information about location.

Differences in the space representation provided by these sources led to the hypothesis of the interaction of two parallel maps underlying cognitive map formation [2]. The first map results from the directional sources processing whereas second one is based only on positional cues. Such concept of two types of space representation was supported by physiological findings provided in [3]. Authors showed that right hippocampus is involved into allocentric map-like representation whereas left hippocampus performs egocentric space representation based on the consecutive choices of movement direction. These representations complement each other supporting different navigational aspects.

In accordance with the abovementioned, an important requirement for navigational behavior models is interaction between egocentric and allocentric spatial representations. This paper focuses on the model of navigational behavior neurodynamics based on original even cyclic inhibitory networks (ECI-networks) [4]. The model concerns two sources of information about space location: so-called path integration [5], linked to self-motion velocity and directional signal processing, and environmental borders.

Path integration is assumed to be performed by grid cells first discovered in the medial entorhinal cortex of freely moving rats [6]. These cells are active whenever rat enters set of locations in the environment, forming hexagonal lattice. Correlates of grid space representation in ECI-network was shown previously in [7, 8]. The main drawback of the navigation based purely on path integration is error accumulation. Spatial information can be corrected by backward connections from place cells or by information about environmental borders. The model focuses on the last alternative.

Two cell types provide information about environmental borders in rat brain: entorhinal border cells, active when animal is located near environmental border [9], and subiculum boundary vector cells, active when the border is situated at the certain distance and allocentric direction from rat [10]. Subiculum has limited projections to the hippocampus [11] whereas entorhinal cortex provides main neocortical input to the hippocampus [12]. For this reason we will focus only on entorhinal border cells.

An algorithm of place cell activity formation in ECInetwork based on weighted summation of grid cell output was published previously in [13]. This paper provides modified algorithm accounting for the interaction between grid and border cells, i.e. interaction between ego- and allocentric representations.

\section{MODEL DESCRIPTION}

\section{A. Spatiotemporal Signal Encoding in ECI-network}

The model under consideration is based on ECI-network. Structural units of the network are called oscillatory modules. Every module consists of two neurons: analogue adaptive and conditional oscillatory neuron, connected via strong recurrent inhibition, whereas modules are connected with each other via weak recurrent inhibition, forming network with several layers (Fig. 1A). Number of layers is preset. All results are presented for 3-layered network but hold for ECI-networks with more layers. Detailed description of ECI-networks is provided in [4, 13].

Network dynamics is described by the following system of nonlinear differential equations:

Research is supported by RFBR, research project № 16-31-00071 mol_a 

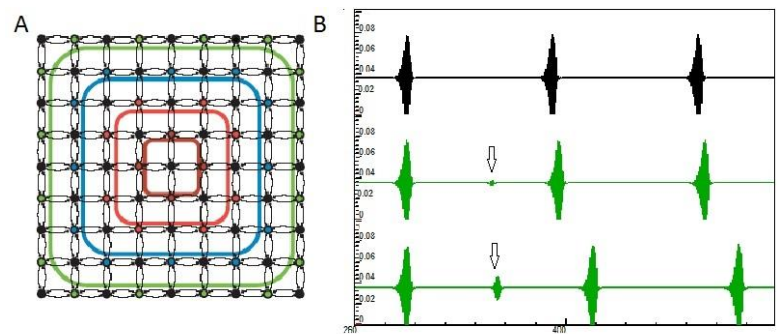

Fig. 1. A. Structure of 4-layered ECI-network. Colored concentric rectangles depict network layers. Colored circles correspond to informational modules; black circles correspond to referent modules. B. Phase shift of the high-frequency oscillations of informational modules (green) relatively oscillations of referent module (black) driven by pulse input signals with different amplitudes. Inputs are applied at the moment depicted by arrows. X- direction: time; Y- direction: membrane potential. All units are dimensionless.

$$
\begin{aligned}
& \tau_{i} \frac{d x_{i}}{d t}=x_{i}-b_{i} z_{i}-\sum_{j=1}^{N} a_{i j} y_{j}+S_{0 i}+S_{i}(t), \\
& T_{i} \frac{d z_{i}}{d t}=-z_{i}+y_{i}, \\
& y_{i}=\left\{\begin{array}{l}
k\left(x_{i}-p_{i}\right), x_{i}>p_{i}, \\
0, x_{i} \leq p_{i},
\end{array}\right. \\
& i=1, \ldots, N,
\end{aligned}
$$

where $N$ - number of neurons, $a_{i j}$ - weight, $S_{i}(t)$ - input signal, $x_{i}$ - membrane potential of the $i$-th neuron, $z_{i}$ - measure of neuron adaptation, $p_{i}-$ threshold, $b_{i}$ - adaptation parameter, $k-$ amplification factor, $T_{i}, \tau_{i}-$ temporal parameters, $y_{i}-$ neuron output, $S_{0 i}$ - permanent input.

Network parameters define variety of dynamical regimes [14]. Hereafter all calculations will be performed with the following values: intramodule weights $\alpha_{i j}=2.27$, weights between modules $\alpha_{i j}=0.001, k=1$; analogue neuron parameters: $S_{0}=0.083, \quad \tau=0.01, \quad T=30, \quad b=10, \quad p=0 ; \quad$ oscillatory neuron parameters: $S_{0}=1, \tau=0,5, T=0.8, b=27, \mathrm{p}=0$. Under these parameters network generates low-frequency rhythm with embedded high-frequency bursts of membrane potential. The ratio of these frequencies allows to treat them as theta and gamma rhythms, respectively. Such cross-frequency interaction of fast (gamma) and slow (theta) frequencies is assumed to underlie information processing in hippocampal formation [1517].

Input signals are applied to the subset of informational modules. Signal patterns can shift phase of gamma-bursts relative endogenous theta-rhythm (Fig. 1B). This shift depends on the amplitude, duration and timing of input. External input, called informational, is specific for each informational module. To quantify phase shift we define a subsystem of referent modules. No signals arrive at referent modules. Referent modules generate time counts with constant frequency similar to hippocampal time cells [18]. Phase shifts of all informational modules relative referent subsystem compose network phase code.

Phase representations of input signals are estimated within the framework of inner dynamical state of the network. Uniform pulse signal, called contextual, is applied to all informational modules in the theta-cycle preceding theta-cycle with informational input. Dependence of gamma-burst starting time on contextual input is summarized in the form of phase response curves (see Fig. 3). Informational input reflects angular velocity signals whereas contextual input reflects linear velocity modulating neuronal activity.

\section{B. Spatial Representation of Place Cells in ECI-network}

Spatial representations in ECI-network are constrained allowing modelling of environmental borders. Details concerning neurodynamic correlates of borders can be found in [19]. Informational modules in ECI-network compose functional ensembles [13]. All ensembles except central are sensitive to the movement direction. This directional ensembles form two clusters encoding clockwise (CW) and counterclockwise $(\mathrm{CCW})$ movement. Central modules form two separate clusters that will encode information about two opposite environmental borders (we will call it northern and southern) (Fig. 2).

Polar coordinates of receptive fields $\left(r_{n}, \varphi_{\mathrm{n}}\right)$ of consequently activated place cells (we will call it mental trajectory) in egocentric system of reference are calculated according to the following algorithm:

$$
\begin{aligned}
& \varphi_{n}=\pi\left(\frac{\sum_{i=1}^{k} a_{i}\left(t_{i n}-t_{r n}\right)+\sum_{j=1}^{m} b_{j}\left(t_{j n}-t_{r n}\right)}{T_{n}\left(\sum_{i=1}^{k} a_{i}+\sum_{j=1}^{m} b_{j}\right)}\right), \\
& r_{n}=\frac{S_{n}-S_{0}}{S_{N}-S_{0}},
\end{aligned}
$$

where $a_{i}$ - weights of informational modules from $\mathrm{CW}$ and CCW - clusters, $b_{j}$ - weights of informational modules from central clusters, $t_{i n}, t_{j n}$ - time of gamma-burst generation of $\mathrm{CW} / \mathrm{CCW}$ and central clusters, respectively, $t_{r n}$ - time of gamma-burst generation of referent modules, $k, m$ - number of informational modules of $\mathrm{CW} / \mathrm{CCW}$-clusters and central clusters respectively, $T_{n}$ - period of referent theta-cycle, $N-$ number of experimental points, $S_{n}$ - amplitude of contextual input at the $n$-th step, $S_{0}$ - initial amplitude of contextual input, $S_{N}$ - final amplitude of contextual input.

This algorithm calculates mental trajectory relative starting position of navigator (starting point of trajectory is always at the center), i.e. egocentric representation. To calculate spatial representations in allocentric system it's necessary to account for starting position and environmental scale. In this case mental trajectory coordinates are calculated as follows:

$$
\begin{aligned}
& \varphi_{n}=\pi\left(\frac{\sum_{i=1}^{k} a_{i}\left(t_{i n}-t_{r n}\right)+\sum_{j=1}^{m} b_{j}\left(t_{j n}-t_{r n}\right)}{T_{n}\left(\sum_{i=1}^{k} a_{i}+\sum_{j=1}^{m} b_{j}\right)}\right), \\
& r_{n}=S_{n} .
\end{aligned}
$$


C. Movement along Borders and at the Center of the Environment
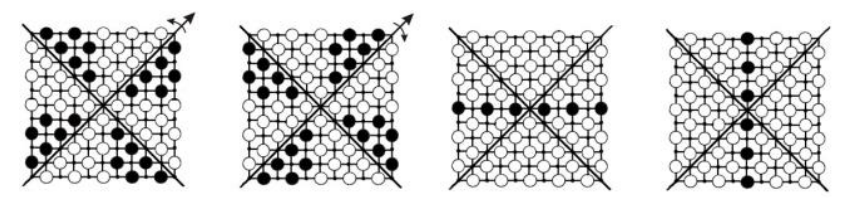

Fig. 2. Clusters of informational modules of 5-layered ECI-network (depicted in black). From left to right: clusters encoding movement in counterclockwise direction (CCW-cluster), clockwise direction (CWcluster), information about environmental borders (southern and northern).

Movement of virtual navigator along borders and at the center of environment is modelled by adjusting respective weights $a_{i}$ and $b_{j}$. Informational modules from $\mathrm{CW}$-cluster have positive weights $a_{i}$ whereas modules from CCW-cluster have negative weights $a_{i}$. Similarly, informational modules of horizontal cluster (north) have positive weights $b_{j}$ and modules of vertical cluster (south) have negative weights $b_{j}$.

Fig. 3A depicts phase response curves for the experiment 3k2fexp2017-bound5.nex. Respective spatial representations in egocentric (normalized space) and allocentric systems of reference in case of different weights are depicted at Fig. 4. Large values of weights $b_{j}$ of informational modules corresponding to one of the borders result in the shift of mental trajectory towards this boundary. In case of large values of weights $b_{j}$ of informational modules encoding information about opposite borders space representation degenerates into straight line corresponding to the movement in narrow environment. Movement at the distance from borders is reflected by large values of $a_{i}$ and low values of $b_{j}$.

Environmental scale is set by the values of contextual input. Larger range of contextual input corresponds to larger scale. Fig. 3B depicts phase response curves for the experiment 3k2fexp2017-bound5-2.nex. Here simulations were held with
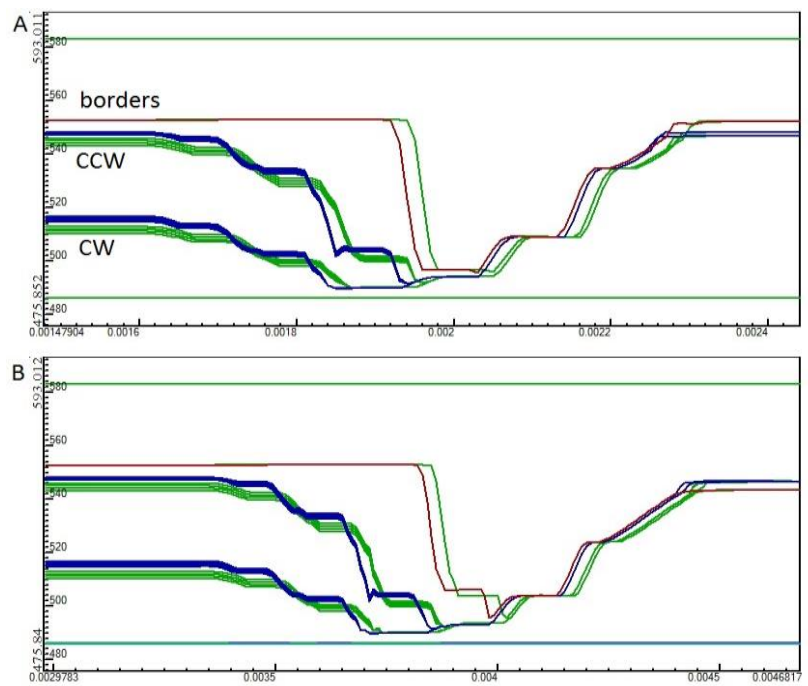

Fig. 3. Phase response curves of the modules of 3-layered ECI-network. Different colors correspond to the informational modules of different layers. Horizontal lines correspond to the referent modules. X-direction: contextual input amplitude. Y-direction: gamma-burst generation time. All units are dimensionless. Informational inputs are applied to the CCWclusters at [352-355], to the CW-clusters at [350.5-353.5], to the border clusters at [356-359]. A. Contextual input is applied at [261-266], amplitude [0.00148-0.00245]. Experiment 3k2fexp2017-bound5.nex. B. Contextual input is applied at [249-254], amplitude [0.00298-0.00468]. Experiment 3k2fexp2017-bound5-2.nex.
A
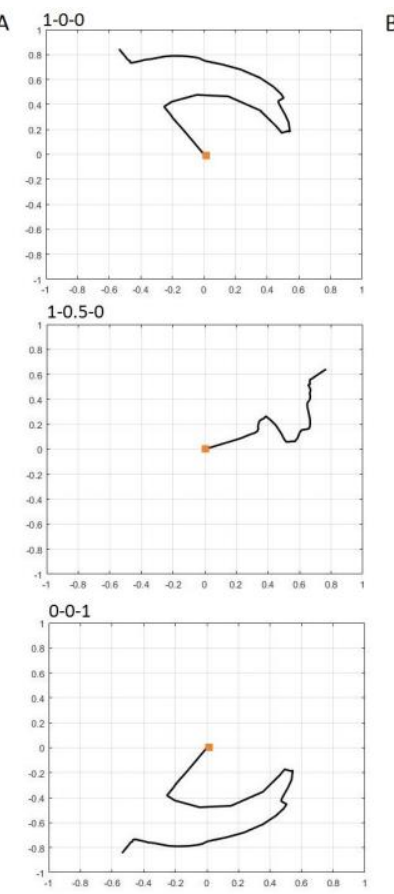

$0-1-0$

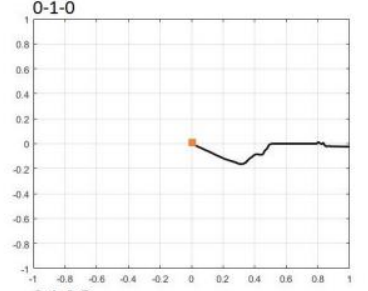

$0-1-0.5$
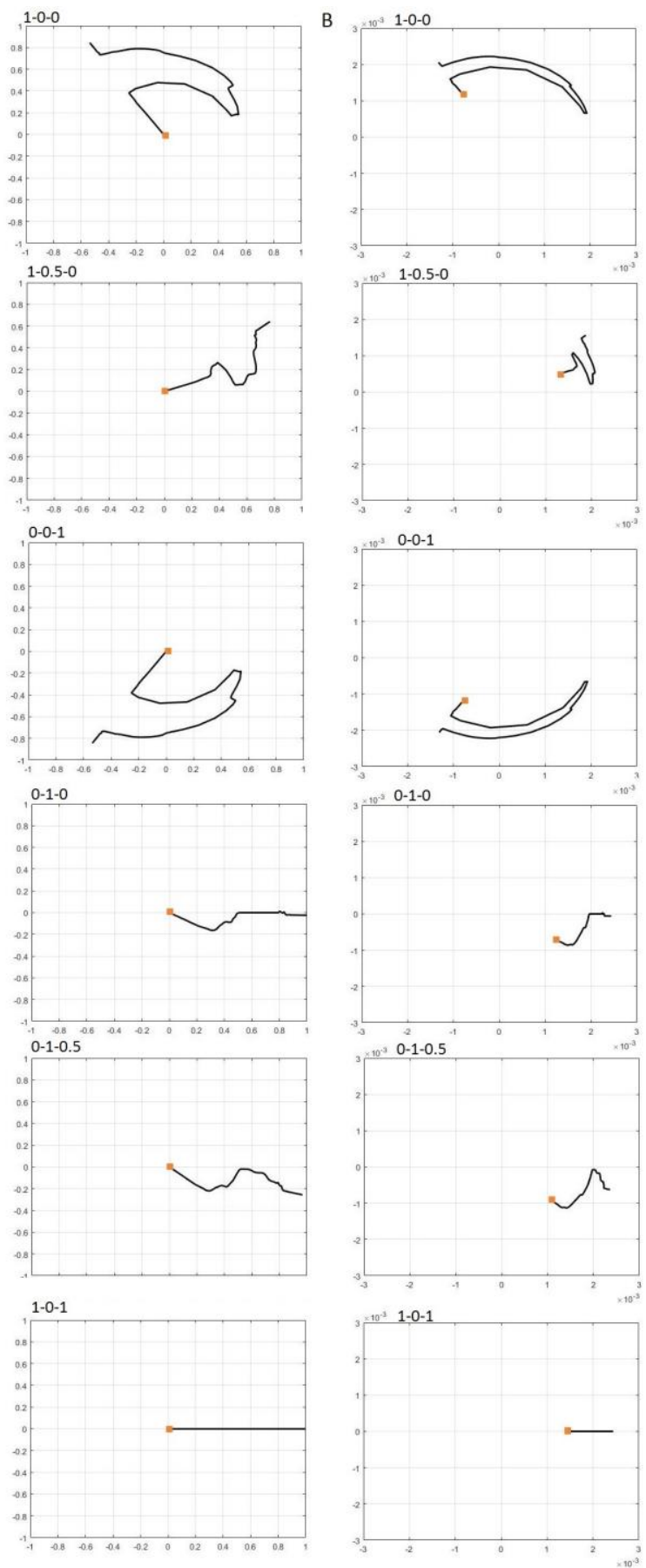

Fig. 4. Spatial representations provided by informational modules of the ECI-network in egocentric (A) and allocentric (B) systems of reference. Initial position of the navigator is marked by orange square. Numbers above representations indicate weights of the informational modules from cluster, corresponding to northern border, CW/CCW clusters (same weight) and cluster corresponding to southern border, respectively. All units are dimensionless. Experiment 3k2fexp2017-bound5.nex.

larger range of contextual input. Respective spatial representation in allocentric system of reference has larger scale than in previous experiment (compare Fig. 4B and Fig. 5) but place cell layout is not affected. 

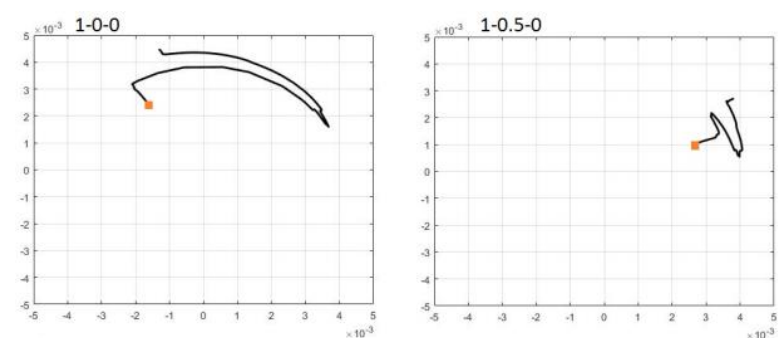

Fig. 5. Spatial representations provided by informational modules of the ECI-network in allocentric system of reference. Initial position of the navigator is marked by orange square. Numbers above representations indicate weights of the informational modules from cluster, corresponding to northern border, $\mathrm{CW} / \mathrm{CCW}$ clusters (same weight) and cluster corresponding to southern border, respectively. All units are dimensionless. Experiment 3k2fexp2017-bound5-2.nex.

\section{DISCUSSION}

There is no agreement about the nature of place cell activity. Entorhinal grid cells are widely assumed to be main input to place cells [20-22]. Other possible candidates are subiculum vector boundary cells [10, 23]. Nevertheless, combination of different sources of spatial information is often claimed to be essential for successful navigation [24, 25].

In the development model, spatial representations of place cell are proposed to result from the interaction between egocentric and allocentric representations provided by grid and border cells, respectively. In line with [26] it is assumed that contribution of grid cell output is more significant for place cells in the center of environment whereas place cell at the periphery rely more on border cell output.

It was shown that stable place cell activity in rat pups is already present from the first postnatal days whereas grid cell activity develops in three weeks [26, 27]. Contribution of border cell activity that develops simultaneously with place cell activity [26] helps to explain this result. In spite of small amount of border cells in medial entorhinal cortex [9], it can be enough to maintain place cell activity at early stages. In addition, interaction between grid and border cells explains why place fields in young rats are more stable at the periphery of environment than at the center (see [26]) at the very early stages when grid cell activity is absent.

Proposed model combines properties of two model types: oscillatory interference and attractor network models (for review see [28]). Here activity pattern arises from oscillations of individual units and is stabilized by units interconnection into network with attractor states. The unique feature of the model is its generality: in one network we have neurodynamic correlates of grid, border, place and time cells. Another model proposed by Welday et al. [29] is worth mentioning. This model tries to explain activity pattern of place, grid and border cells, but not time cells. Moreover, it does not answer a question about interactions between these types of cells

Madl et al. [30] also exploit the idea of grid and border cells interaction to simulate place cell activity. This model focuses on the interaction between cell types but does not explain how grid and border cell activity arises. There information from these two types of cells is combined according to Bayesian rule: weight coefficient are assigned depending on reliability of the information.
In the development model weights depend on the proximity of borders. Movement along one of the borders is reflected in high values of the weights of informational units from corresponding cluster, whereas movement at the center corresponds to high values of the weights of $\mathrm{CW}$ and $\mathrm{CCW}$ clusters. Changing weights also allows to modulate shape of the environment. Large values of weights of informational units corresponding to opposite borders reflect proximity of both borders and are used to simulate movement in narrow space.

Changing in the environment scale in the model simulated by different amplitude ranges of contextual input did not affect sequence of place cell firing. This result is also in agreement with physiological findings provided in [31]. It was shown that fraction of the recorded place fields, but not all, scaled with the scaling of environment: fields stayed at the same angular position and the same relative radial position. Stretching of the place cell layout in linear space was also shown in [32]. Proposed model together with these results support the idea of topological representation of space provided by hippocampus (see [33, 34]).

Interaction of border and grid cell outputs does not account for all properties of place cells. In particular, it can not explain remapping of place cell layout due to task [35], fear [36], internal state of the rat [37] and other factors. Therefore, further development of the model will focus on the modelling of the effect of non-spatial context on place cell firing.

\section{REFERENCES}

[1] J. O'Keefe and J. Dostrovsky. "The hippocampus as a spatial map. Preliminary evidence from unit activity in the freely moving rat". Brain Res., 1971, vol. 34, pp. 171-175.

[2] L.F. Jacobs and F. Schenk. "Unpacking the cognitive map: the parallel map theory of hippocampal function”. Psychol. Rev., 2003, vol. 110, № 2, pp. 285-315.

[3] K. Iglói, C.F. Doeller, A. Berthoz, L. Rondi-Reig, and N. Burgess. "Lateralized human hippocampal activity predicts navigation based on sequence or place memory”. PNAS USA, 2010, vol. 107, № 32, pp. 14466-14471.

[4] V.D. Tsukerman. "Nonliner dynamics of sensory cognition, or What and how does the brain encode". Rostov State University Press, Rostov-onDon, 2005. (in Russian)

[5] A.S. Etienne and K.J. Jeffery. "Path Integration in Mammals". Hippocampus, 2004, vol. 14, pp. 180-192

[6] T. Hafting., M. Fyhn, S. Molden, M.B. Moser, and E.I. Moser. "Microstructure of a spatial map in the entorhinal cortex". Nature, 2005 , vol. 436, pp. 801-806.

[7] V.D. Tsukerman, Z.S. Eremenko, O.V. Karimova, S.V. Kulakov, and A.A. Sazikin. "Mathematical model of hippocampal space encoding. I. Grid cell neurodynamics”. Math. Biol. Bionif., 2012, vol. 7, № 1, pp. 206-243. (in Russian)

[8] Z.S. Kharybina. "A model of neurodynamics of hippocampal formation neurons performing spatial processing based on even cyclic inhibitory networks". In Biologically Inspired Cognitive Architectures (BICA) for Young Scientists. Proceedings of the First International Early Research Career Enhancement School (FIERCES 2016). Advances in Intelligen Systems and Computing, vol. 449. A.V. Samsonovich, V.V. Klimov, and G.V. Rybina. Springer, 2016, pp. 79-84.

[9] T. Solstad, C.N. Boccara, E. Kropff, M.B. Moser, and E.I. Moser "Representation of geometric borders in the entorhinal cortex". Science, 2008, vol. 322, pp. 1865-1868.

[10] C. Lever, S. Burton, A. Jeewajee, J. O'Keefe, and N. Burgess. "Boundary vector cells in the subiculum of the hippocampal formation". J. Neurosci., 2009, vol. 29, pp. 9771-9777. 
[11] M.P. Witter, F.G. Wouterlood, P.A. Naber, and T. Van Haeften. "Anatomical organization of the parahippocampal-hippocampal network". Ann. NY Acad. Sci., 2000, vol. 911, pp. 1-24.

[12] D. Amaral and P. Lavenex. "Hippocampal Neuroanatomy". in P. Andersen, R. Morris, D. Amaral., T. Bliss, and J. O'Keefe. The Hippocampus Book. Oxford University Press, 2007, pp. 37-114.

[13] Z.S. Kharybina, V.D. Tsukerman, and S.V. Kulakov. "Mathematical model of the microcircuit organization of freely scalable ECI-network as a former of spatial processing in hippocampal-entorhinal brain system". Applied Mathematical Sciences, 2014, vol. 8, № 12, pp. 549-572.

[14] Z.S. Kharybina. "Mechanisms of phase synchronization in neural even cyclic inhibitory networks”. Biophysics, 2016, vol. 61, № 3, pp. 485493.

[15] J.J Chrobak and G. Buzsáki. "High-frequency oscillations in the output networks of the hippocampal-entorhinal axis of the freely-behaving rat". J. Neurosci., 1996, vol. 16, № 9, pp. 3056-3066.

[16] L.L. Colgin, T. Denninger, M. Fyhn, T. Hafting, T. Bonnevie, O. Jensen, M.B. Moser, and E.I. Moser. "Frequency of gamma oscillations routes flow of information in the hippocampus". Nature, 2009, vol. 462, pp. 353-357.

[17] L.L. Colgin and E.I. Moser. "Gamma oscillations in the hippocampus". Physiology, 2010, vol. 25, № 5, pp. 319-329.

[18] B.J. Kraus, R.J. Robinson, J.A. White, H. Eichenbaum, M.E. Hasselmo. "Hippocampal time cells: time versus path integration". Neuron, 2013, vol. 78, № 6, pp. 1090-101.

[19] Z.S. Kharybina. "The effect of environmental borders on spatial representation of place cells in the model of spatial navigation neurodynamics based on even cyclic inhibitory networks". In the proceedings of the XIX International conference Neuroinformatics2017, vol. 2, pp. 21-29. (in Russian)

[20] J. O'Keefe and N. Burgess. "Dual phase and rate coding in hippocampal place cells: theoretical significance and relationship to entorhinal grid cells". Hippocampus, 2005, vol. 15, pp. 853-866.

[21] M.C. Fuhs and D.S. Touretzky. "A spin glass model of path integration in rat medial entorhinal cortex". J. Neurosci., 2006, vol. 26, pp. 42664276.

[22] E.T. Rolls, S.M. Stringer, and T. Elliot. "Entorhinal cortex grid cells can map to hippocampal place cells by competitive learning". Network, 2006, vol. 17, pp. 447-465.

[23] C. Barry, C. Lever, R. Hayman., T. Hartley, S. Burton, J. O'Keefe, K. Jeffery, and N. Burgess. "The boundary vector cell model of place cell firing and spatial memory". Rev. in the Neurosci., 2006, vol. 17, pp. $71-97$.
[24] E. Save, V. Hok, S. Renaudineau, C. Parron, and B. Poucet. "Cue and goal encoding in rodents: a source of inspiration for robotics?" In Robotics and cognitive approaches to spatial mapping. STAR. M.E. Jefferies, W.-K. Yeap eds., (Berlin, Heidelberg. Springer-Verlag), 2008, vol. 38, pp. 163-180.

[25] E. Save and B. Poucet. "Involvement of the hippocampus and associative parietal cortex in the use of proximal and distal landmarks for navigation". Behav. Brain Res., 2000, vol. 109, pp. 195-206.

[26] T.L. Bjerknes, E.I. Moser, and M.B. Moser. "Representation of the geometric borders in the developing rat". Neuron, 2014, vol. 82, pp. 7178.

[27] T.J. Wills., F. Cacucci, N. Burgess, and J. O'Keefe. "Development of the hippocampal cognitive map in preweanling rats". Science, 2010, vol. 328, pp. 1573-1576

[28] Z. Navratilova, LM. Giocomo, J.M. Fellous, M.E. Hasselmo, and B.L. McNaughton. "Phase precession and variable spatial scaling in a periodic attractor map model of medial entorhinal grid cells with realistic after-spike dynamics”. Hippocampus, 2012, vol. 22, № 4, pp. 772-89.

[29] A.C. Welday, I.G. Shlifer, M.L. Bloom, K. Zhang, and H.T. Blair "Cosine directional tuning of theta cell burst frequencies: evidence for spatial coding by oscillatory interference". J. Neurosci., 2011, vol. 31, № 45, pp. 16157-16176.

[30] T. Madl, S. Franklin, K. Chen, D. Montaldi, and R. Trappl. "Bayesian integration of information in hippocampal place cells". Plose One, 2014, vol. 9, № 3, e89762.

[31] R.U. Muller and J.L. Kubie. "The effects of changes in the environment on the spatial firing of hippocampal complex.-spike cells". J. Neurosci., 1987, vol. 7, № 7, pp. 1951-1966.

[32] K. Diba and G. Buzsaki. "Hippocampal network dynamics constrain the time lag between pyramidal cells across modified environments". J. Neurosci, 2008, vol. 28, № 50, pp. 13448-13456.

[33] Y. Dabaghian, F. Memoli, L. Frank., and G. Carlsson. "A topological paradigm of hippocampal spatial map formation using persistent gemology”. PLOS Comput. Biol., 2012, vol. 8, № 8, e1002581.

[34] A. Babichev, D. Ji, F. Memoli, and Y. Dabaghian. "A topological model of the hippocampal cell assembly network". Front. Comput. Neurosci., 2016, vol.10, 50.

[35] E.J. Markus, Y.L. Qin, B. Leonard, W.E. Skaggs, B.L. McNaughton, and C.A. Barnes. "Interactions between location and task affect the spatial and directional firing of hippocampal neurons". J. Neurosci., 1995, vol. 15, pp. 7079-7094.

[36] M.A.P. Moita, S. Rosis, Y. Zhou, J.E. LeDoux, and H.Y. Blair. "Putting fear in its place: remapping of hippocampal place cells during fear conditioning”. J Neurosci., 2004, vol. 24, № 31. pp. 7015-7023.

[37] E.R. Wood, P.A. Dudchenko, R.J. Robitsek, and H. Eichenbaum. "Hippocampal neurons encode information about different types of memory episodes occurring in the same location". Neuron, 2000, vol. 27, pp. 623-633. 